\title{
IN SILICO MODELING OF MONOMERIC DEXAMETHASONE-INDUCED RAS-RELATED PROTEIN 1 AND RAS HOMOLOG ENRICHED IN STRIATUM: ROLE OF N TERMINUS AND STRUCTURE-FUNCTION RELATIONSHIP
}

\author{
RASHMI VERMA ${ }^{1}$, NAVIN KUMAR ${ }^{2}$, ASHISH THAPLIYAL ${ }^{1 *}$
}

${ }^{1}$ Department of Life Sciences, Graphic Era (Deemed to be University), Dehradun, Uttarakhand, India. ${ }^{2}$ Department of Biotechnology, Graphic Era (Deemed to be University), Dehradun, Uttarakhand, India. Email: ashish.thapliyal@geu.ac.in

Received: 13 July 2018, Revised and Accepted: 28 August 2018

\begin{abstract}
Objective: Dexamethasone-induced Ras-related protein 1 (Dexras1) and Ras homolog enriched in striatum (RHES) are the two monomeric small G proteins that belong to Ras superfamily. These two proteins show $62 \%$ similarity. Both of these proteins are involved in signaling and modulation of several pathophysiological processes. They have unique GTP binding domain and a unique $\mathrm{C}$ and $\mathrm{N}$ terminus. C terminus is known to interact with several proteins; however, the role of its unique $\mathrm{N}$ terminus is still not known. The three-dimensional (3D) structure of these proteins is also not available in any of the databases yet. This present study approaches bioinformatics tools and servers to predict the 3D structure of these two proteins in silico.
\end{abstract}

Methods: In this study, two bioinformatics servers were used, namely Swiss modeling server and Iterative Threading ASSEmbly Refinement (I-TASSER) server.

Results: Both servers developed many alignment templates of Dexras1 and RHES. These alignments were used to develop 3D structure using Pymol. These models have different regions of proteins such as $\mathrm{N}$ terminus, GTP-binding domains, effector loop, $\mathrm{C}$ terminus, and the unique CAAX site. The models deduce that the $\mathrm{N}$-terminals of both Dexras1 and RHES are unique regions that might possible be dangling out of the protein while it gets inserted into the membrane. We hypothesize that this unique $\mathrm{N}$-terminal might have a distinct role in the modulation of $\mathrm{N}$-type calcium channels.

Conclusion: All the models generated show predicted 3D structure of Dexras1 and RHES protein. This study of structural prediction will be helpful in knowing the interaction of Dexras1 and RHES and a step forward to target these two proteins as a novel therapeutic drug.

Keywords: G proteins, Homology modeling, Dexamethasone-induced Ras-related protein 1, Ras homolog enriched in striatum, Pathophysiological processes, Structure-function relationship, Iterative Threading ASSEmbly Refinement, Swiss server.

(C) 2019 The Authors. Published by Innovare Academic Sciences Pvt Ltd. This is an open accessarticle under the CC BY license (http://creativecommons. org/licenses/by/4. 0/) DOI: http://dx.doi.org/10.22159/ajpcr.2019.v12i1.28486

\section{INTRODUCTION}

Dexamethasone-induced Ras-related protein 1 (Dexras1) (also known as AGS1 or Rasd1) and Ras homolog enriched in striatum (RHES) (also known as Rasd2) are the monomeric G proteins, which are involved in many pathophysiological processes [1]. Expression of Dexras1 (Rasd1 and formerly called as Activators of G-protein Signaling 1) is upregulated by dexamethasone and estriadiol and is expressed in many tissues [2-5]. Another monomeric closely resembling Dexras1 is (RHES, Rasd2) and it bears about 62\% similarity to Dexras1 [6]. Both these monomeric Ras proteins show about $35 \%$ similarities to other members of Ras proteins $[7,8]$. They have similar GTP binding sites like all Ras proteins, but the $\mathrm{C}$ and $\mathrm{N}$ terminus are unique. Human Dexras1 is a $30 \mathrm{kDa}$ protein, 281 amino acids expressed in almost all tissue, whereas RHES is a protein with 266 amino acids and is primarily expressed in the brain and its expression is regulated by thyroid hormones. Both Dexras1 and RHES have been implicated in many signaling pathways [1] and have three prominent structural domains/conserved regions: (i) Four highly conserved GTP binding domains, (ii) Effector loop - might possibly participate in protein-protein interactions with other signaling molecules and is necessary for full biological activity, and (iii) A CAAX sequence at the end of C-terminal - a consensus site for isoprenylation CVIS [9]. All these conserved regions are thought to be important for the activity of Dexras1. Dexras1 has similar signaling properties as RHES in terms of modulation of N-type calcium channel Cav2.2 [10]. Their role in cardiovascular diseases, Huntington disease, regulation of rhythms, neurotransmitter-mediated behavior, and cancer has also been investigated [11-14].
The enormous range of activity that showed by the two monomeric proteins, Dexras1 and RHES, may involve some of their structural determinants/ domains, that might assist them in performing a wide range of functions. These monomeric Ras proteins have a unique $\mathrm{C}$ and $\mathrm{N}$-terminal. The C-terminal has been shown to interact with proteins such as neuronal nitric oxide synthase (nNOS - stimulated by NMDA receptors) and PTB domain toward N-terminal of CAPON [15]. They suggested that the formation of trimeric complex between Dexras1, nNOS, and CAPON leads to S-nitrosylation of cysteine at $11^{\text {th }}$ position (cys 11), resulting in an increased guanine-nucleotide exchange. Many other studies so far have implicated that C-terminal interactions and s-nitrosylation of cystein 11 are prominent strategies for the modulation of different signalling pathways. However, questions such as what is the role of unique $\mathrm{N}$-terminal region of Dexras1 in the modulation of N-type calcium ion channels (Cav2.2) still remain. Most of the studies carried out until date focus on investigating modulation of signaling pathways by Dexras1 and RHES. Although the general framework of different domains has been illustrated by Graham et al. 2002, to date, the role of N-terminal of Dexras1 and RHES in the modulation of voltage-gated Cav2.2 or any other interacting protein has not been investigated.

As the function(s) of its N-terminal of Dexras1 and RHES are still unknown, so to predict the interaction of $\mathrm{N}$-terminal, we applied an in-silico approach. Although the sequences of both proteins are known and the GTP-binding domains present in the core of protein well marked, we still do not understand the molecular mechanism of these two monomeric proteins by which they modulate different physiological mechanisms. 


Dexras1

MODEL1 of Dexras1, Template 4efm. 1.A, Seq Identity Coverage 38.04\%, Description GTPase HRas, Oligostate: Monomer, Ligands None, GMQE 0.40, QMEAN4-4.82,

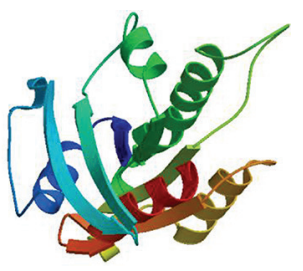

MODEL2 of Dexras1, Template 2ery. 1.A, Seq Identity Coverage 40.24\%, Description Ras-related protein R-Ras2, Oligostate: Monomer, Ligands None, GMQE 0.40, QMEAN4-4.17,

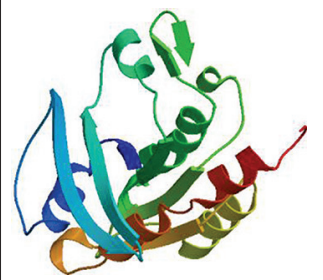

MODEL3 of Dexras1, Template 4dst. 1.A, Seq Identity Coverage 38.73\%, Description GTPase KRas, isoform 2B, Oligostate: Monomer, Ligands None, GMQE 0.40, QMEAN4-7.19,

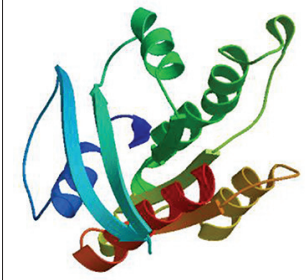

MODEL4 of Dexras1, Template 3rap. 1.B, Seq Identity Coverage 39.88\%, Description PROTEIN (G protein RAP2A), Oligostate: Monomer (matching prediction), Ligands None, GMQE 0.42, QMEAN4-3.94,

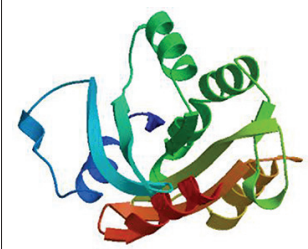

MODEL5 of Dexras1, Template 2gf0.1.A, Seq Identity Coverage 43.21\%, Description GTP-binding protein Di-Ras1, Oligostate: Monomer, Ligands None, GMQE 0.43, QMEAN4-4.38,

\section{RHES}

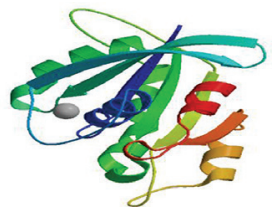

MODEL1 of RHES, Template 3kko. 1.A, Seq Identity Coverage $40.36 \%$ Description Ras-related protein M-Ras, Oligostate: Monomer, Ligands 1 x MG, GMQE 0.41, QMEAN4-4.22,

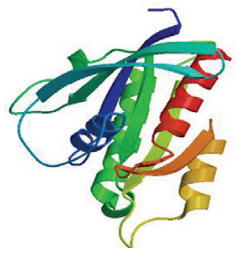

MODEL2 of RHES, Template 2fn4.1.A, Seq Identity Coverage 42.01\%Description Ras-related protein R-Ras, Oligostate: Monomer, Ligands None, GMQE 0.44, QMEAN4-4.03,

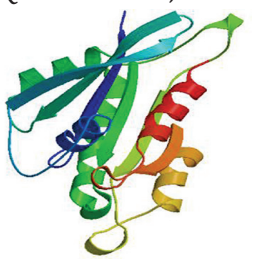

MODEL3 of RHES, Template 2erx. 1.A, Seq Identity Coverage 45.45\%Description GTP-binding protein Di-Ras2, Oligostate: Monomer, Ligands None, GMQE 0.47, QMEAN4-2.35

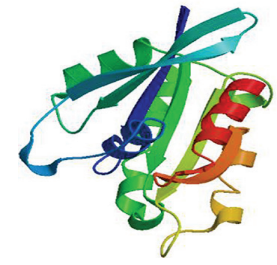

MODEL4 of RHES, Template 2gf0.1.A, Seq Identity Coverage $46.91 \%$ Description GTP-binding protein Di-Ras1, Oligostate: Monomer, Ligands None, GMQE 0.46, QMEAN4-3.59

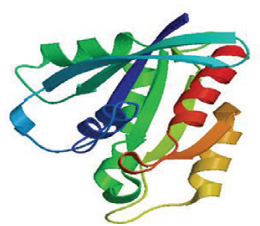

MODEL5 of RHES, Template 4tqa. 1.A, Seq Identity Coverage 39.02\%Description GTPase KRas, Oligostate: Monomer, Ligands None, GMQE 0.47, QMEAN4-3.68

Fig. 1: Models of Dexamethasone-induced Ras-related protein 1 and Ras homolog enriched in striatum generated by Swiss homology modeling server

The in silico approach for the modeling of different chemicals and their structural analysis has been reported by many researchers. Sahare and Moon [16] used in silico strategies to find ligand binding efficiencies of the active site of PBP4 toward selected $\beta$-lactam antibiotics as well as phytochemicals. They reported the highest binding affinity to the active site pocket of PBP4 of $\beta$-lactam resistant Enterococcus faecalis isolated from uropathological samples of urinary tract-infected patients. This study could be useful in designing more effective phytoligandbased therapeutic antibacterial compounds against PBP4 of E. faecalis.
Another researcher, Gupta et al. [17] also used bioinformatics tools to reveal the secondary structure features and characterization of an enzyme SOD and its different isoforms which might be beneficial for oxidative stress management in plants during germination phases. In our study, we also have used different in silico approaches. As the experimentally determined crystal structure of the two abovementioned proteins (Dexras1 and RHES) is not available, so with the help of bioinformatics tools, we have tried to develop a hypothesis about the unique three-dimensional (3D) structure of these proteins and also 


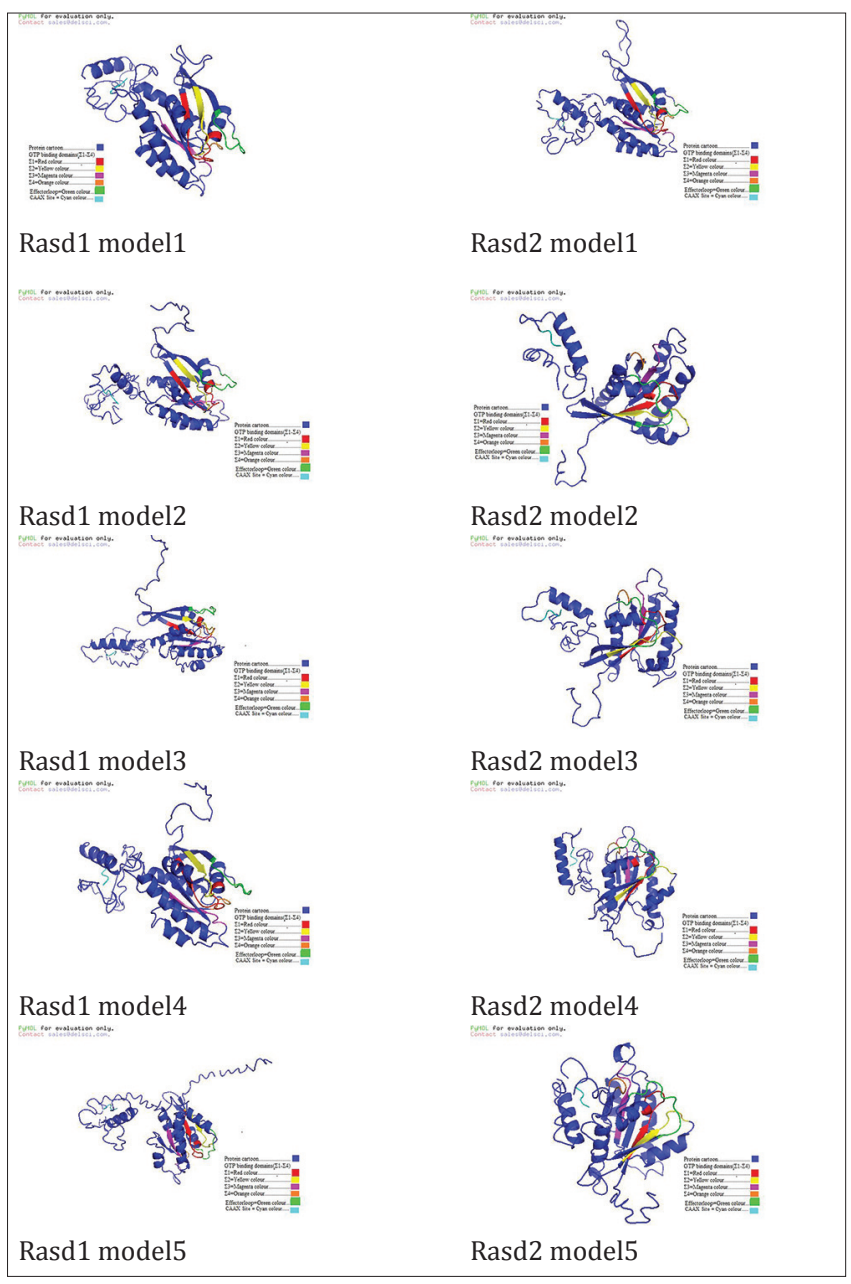

Fig. 2: Models of dexamethasone-induced Ras-related protein 1/ Rasd1 and Ras homolog enriched in striatum/Rasd2 generated by Iterative Threading ASSEmbly Refinement server using Pymol software

tried that the models obtained by bioinformatics tools might provide some information about the structure and interaction of these proteins.

\section{MATERIALS AND METHODS}

\section{Experimental procedures}

On the basis of the previous study [10], it has been reported that these two proteins are involved in modulation of $\mathrm{N}$-type calcium channels. Protein sequences of human Dexras1 (Accession number: AF498923) and RHES (Accession number BC013419) were retrieved from the National Center for Biotechnology Information and queried in FASTA format.

Homology modeling is an approach to construct the atomic-resolution model of the "target" protein from its amino acid sequence. The method produces experimental 3D structure of a related homologous protein (the "template") which relies on the identification of one or more known protein structures likely to resemble the structure of the query sequence. Moreover, this approach is also based on the production of an alignment that maps residues in the query sequence to residues in the template sequence to produce homologous 3D structure of the experimental protein [18]. The homology modeling procedure can be broken down into four sequential steps: Template selection, targettemplate alignment, model construction, and model assessment [19].

There are a number of free servers that create homology models (also called comparative models) for a submitted amino acid sequence or that offer libraries of 3D models created in advance for protein sequences.
In the present study, we have used two homology modeling servers, namely Swiss homology modeling and Iterative Threading ASSEmbly Refinement (I-TASSER) server, to predict the 3D structure of the proteins.

\section{Method}

Swiss model server [20] is a server for automated comparative modeling of 3D protein structures. All homology-modeling methods consist of the following four steps: (i) Template selection, (ii) targettemplate alignment, (iii) model building, and (iv) evaluation. Energy minimization or molecular dynamic methods are in general not able to improve the accuracy of the models and are used in Swiss model only to regularize the structure.

Our query for Dexras1 protein homology modeling by Swiss server generated several templates and we developed five models based on five templates, namely, (a) GTPase HRas (pdb ID 4efm.1.A, identity 38.04\%), (b) Ras-related protein R-Ras2 (pdb ID - 2ery.1.A, identity 40.24\%), (c) GTPase KRas, isoform 2B (pdb ID - 4dst.1.A, identity 38.73\%), (d) PROTEIN - G protein RAP2A (pdb ID 3rap.1.B, identity 39.88\%), and (e) GTP-binding protein Di-Ras1 (pdb ID - 2gf0.1.A, identity 43.21\%); Similar approach was also used for RHES and our query generated several templates and we developed five models based on five templates, namely, (a) Ras-related protein M-Ras (pdb ID - 3kko.1.A, identity 40.36\%), (b) Ras related protein R-Ras (pdb ID - 2fn4.1.A, identity 42.01\%), (c) GTP-binding protein Di-Ras2 (pdb ID - 2erx.1.A, identity $45.45 \%$ ), (d) GTP-binding protein Di-Ras1 (pdb ID 2gf0.1.A, identity 46.91\%), and (e) GTPase KRas (pdb ID 4tqa.1.A, identity 39.02\%).

The second approach was based on the I-TASSER Server. It is an online server built in the Yang Zhang Lab at the University of Michigan, Ann Arbor, allowing users to submit sequences and obtain structure and function predictions. (Server link - http://zhanglab.ccmb.med.umich. edu/I-TASSER/, Zhang-server, University of Michigan, USA). I-Tasser server uses slightly different approaches to build protein models. In general, Swiss server produces homology protein structures on the bases of template alignment, while in I-Tasser server, full-length structure models are constructed by reassembling structural fragments from threading templates.

\section{I-TASSER server}

I-TASSER is a bioinformatics method for predicting 3D structure model of protein molecules from amino acid sequences [21]. It detects structure templates from the Protein Data Bank by a technique called fold recognition (or threading). The full-length structure models are constructed by reassembling structural fragments from threading templates using replica exchange Monte Carlo simulations [21].

I-TASSER has been extended for structure-based protein function predictions, which provides annotations on ligand binding site, gene ontology, and enzyme commission by structurally matching structural models of the target protein to the known proteins in protein function databases [22].

This server uses multiple-threading alignments by local metathreading-server and iterative template fragment assembly simulations and the function insights are derived by matching the 3D models with BioLiP protein function database. The query for the structure of Dexras1 and RHES (same accessions numbers) was submitted to the server, and we received the detailed coordinates for five models each of Dexras1 and RHES. This coordinate file was converted into an pdb file, and details of the 3D conformations were generated and labeled using PYMOL (The PyMOL Molecular Graphics System, Version 1.5.0.4 Schrödinger, LLC.), while the free energies for each structure were computed using SPDBV (http://www.expasy.org/spdbv/). The validation of the structures has been done using The Structure Analysis and Verification Server version 4. This meta server runs seven programs named as PROCHECK, WHAT_CHECK, ERRAT, VERIFY 3D, PROVE CRYST1 record matches, Ramachandran Plot, WedMol Viewer 


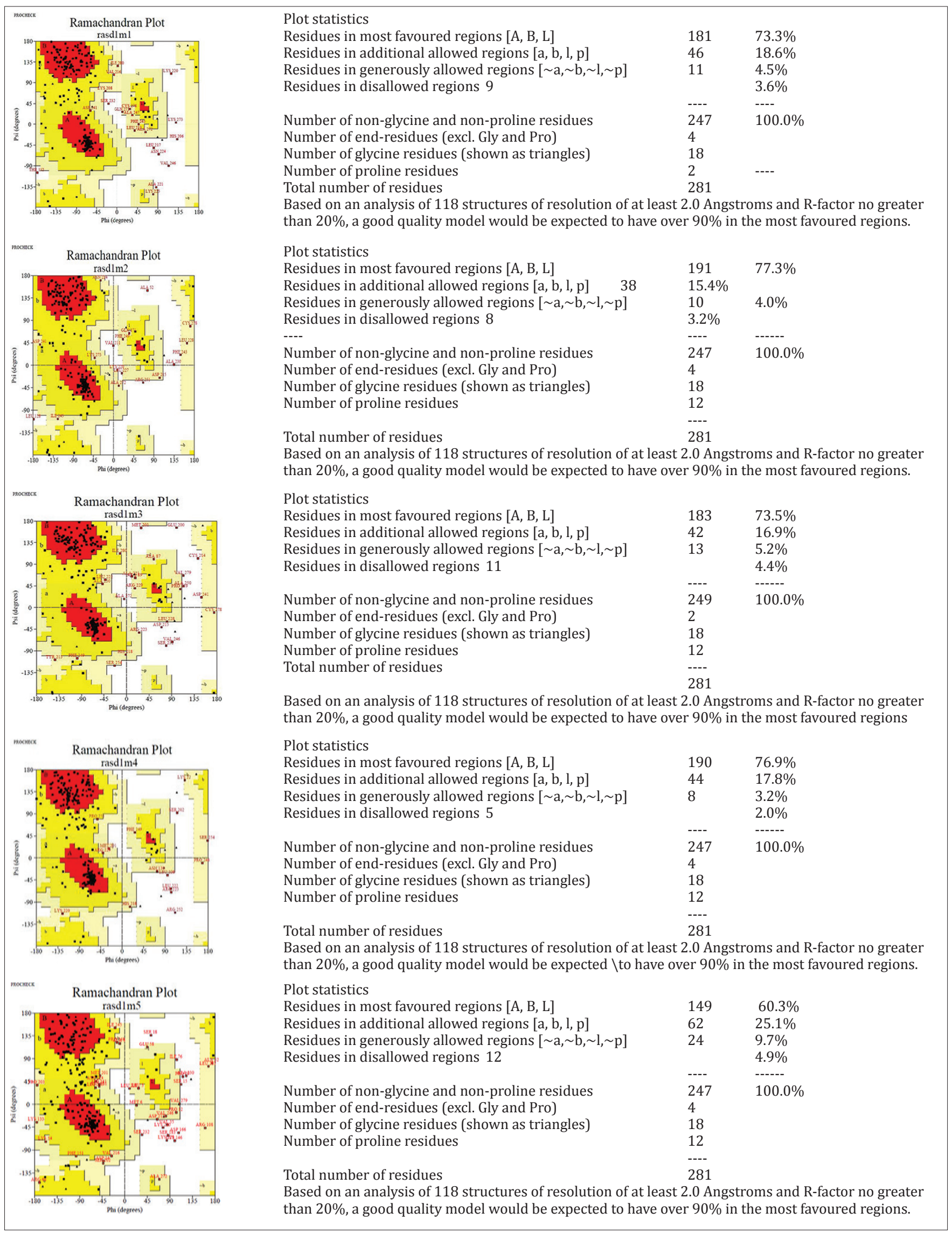

Fig. 3: Ramachandran plots of the five models (developed by Iterative Threading ASSEmbly Refinement) of dexamethasone-induced Ras-related protein 1/Rasd1 using "The Structure Analysis and Verification Server version 4" 


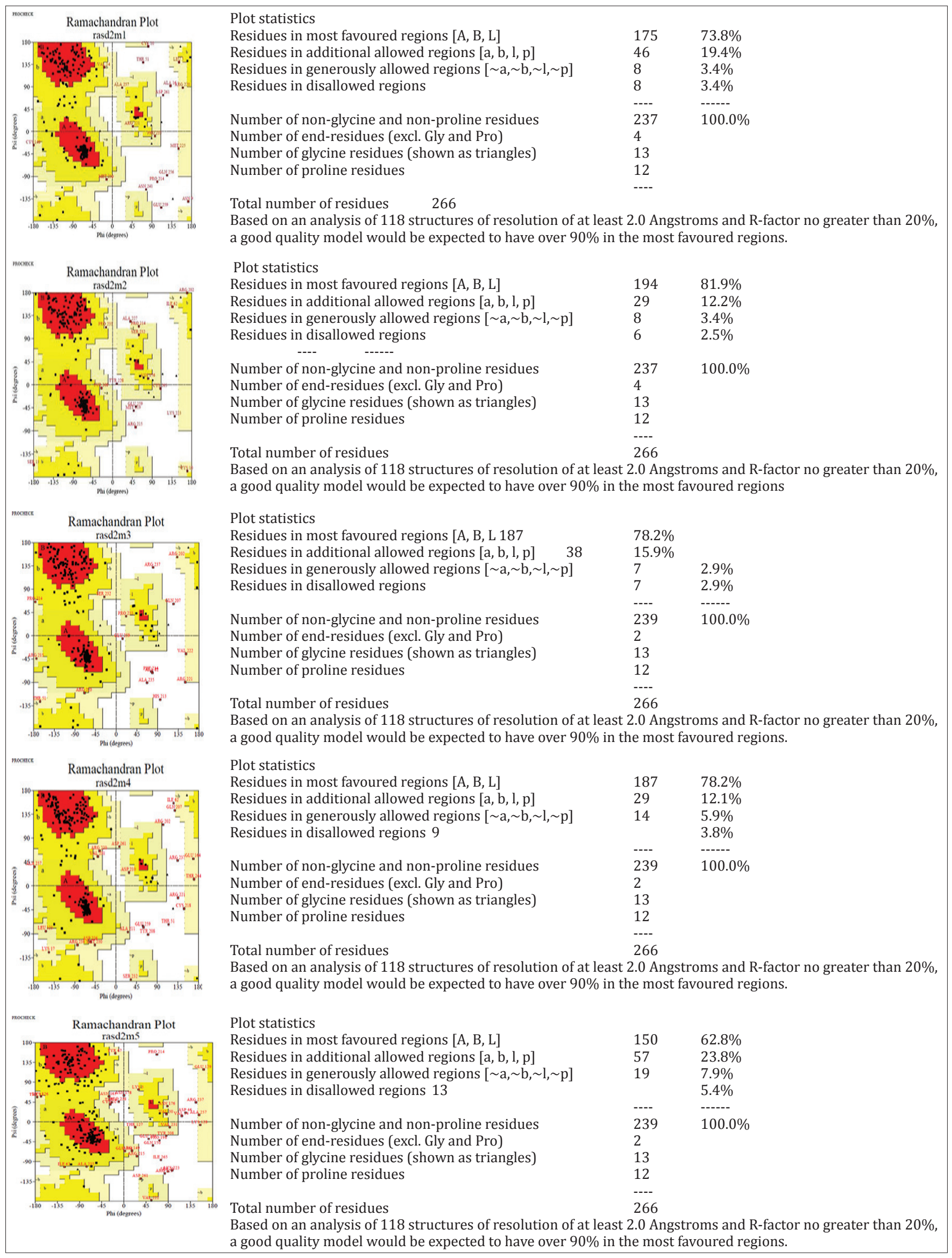

Fig. 4: Ramachandran plots of the five models (developed by Iterative Threading ASSEmbly Refinement) of Ras homolog enriched in striatum/Rasd2 using "The Structure Analysis and Verification Server version 4" 
(link http://nihserver.mbi.ucla.edu/SAVES) for checking and validating protein structures during and after model refinement.

\section{RESULTS}

Homology models for the structural prediction of Dexras1 and RHES have been done using two different homology modeling servers:

First, structural model of Dexras1 and RHES was done using homology modeling by Swiss server. In this approach, the sequences of dexras1 and RHES were run on Swiss server. This server developed different models based on the template structures available in the server. The models generated using this approach are represented in a panel of figures in Fig. 1 for Dexras1and RHES. All these models generated showing 38-46 \% identity with the existing protein models.

The sequences were also submitted to I-TASSER SERVER. This server computed relative positions of amino acid and developed a model, and it also integrated the known templates and their probable structures. This server also computed five different hypothetical models and calculates their lowest free energy states also. The models developed using this server are represented in Fig. 2 which include rasd1 $\mathrm{m} 1$, $\operatorname{rasd} 2 \mathrm{~m} 2, \operatorname{rasd} 3 \mathrm{~m} 3$, rasd $4 \mathrm{~m} 4$, and rasd5m5 for Dexras1/Rasd1 and $\operatorname{rasd} 2 \mathrm{~m} 1, \operatorname{rasd} 2 \mathrm{~m} 2, \operatorname{rasd} 2 \mathrm{~m} 3, \operatorname{rasd} 2 \mathrm{~m} 4$, and $\operatorname{rasd} 2 \mathrm{~m} 5$ for RHES/Rasd2

These models deduce that the N-terminals of both Dexras1 and RHES are unique regions that might possible be dangling out of the protein (non-random domains), while it gets inserted into the membrane. We hypothesize that this unique $\mathrm{N}$-terminal might have a distinct role in the modulation of $\mathrm{N}$-type calcium channels.

\section{Validation of the structures}

The structures generated after using I-Tasser server were validated using "The Structure Analysis and Verification Server version 4." This meta server runs different programs named as PROCHECK, WHAT_CHECK, ERRAT, VERIFY 3D, PROVE, CRYST1 record matches, Ramachandran Plot, and WedMol Viewer (link http://nihserver.mbi. Ucla.edu/SAVES), for checking and validating protein structures during and after model refinement. The results generated for all the programs have been saved in Pdf files, which includes prove plots, Ramachandran plot of all residues, ProCheck PDF files, and a verification plot, for all the models of the two proteins. The Ramachandran plots generated for all the models are represented in Fig. 3 for Dexras1/Rasd1 and Fig. 4 for RHES/ Rasd2, showing some of the residues falls under disallowed region in the analysis of Ramachandran Plot, whereas most of the residues falls under most favored regions together with allowed regions.

Simultaneously, new validated pdb version has been obtained using the Structure Validation and Quality server MolProbity (link: http:// molprobity.biochem.duke.edu/) for structure validation on clientuploaded or PDB ID-specified files, using all-atom contact analysis tools and updated geometrical criteria for phi/psi, sidechain rotamer, and Cbeta deviation.

Link http://www.rcsb.org/pdb/static.do?p=software/software_links/ analysis_and_verification.html.

\section{DISCUSSION}

Dexras1 and RHES, both proteins are involved in modulation of many physiological processes, but the 3D structure and the exact mechanism of modulation involved are not known yet. In the present study, in-silico approach has been used to predict the 3D structure of these proteins which might be helpful in prediction of probable mechanisms of signaling involved in different physiological processes. Our present study is a step forward in understanding the structure-function relationship of Dexras1 with special reference to modulation of $\mathrm{N}$-type calcium channels.

Our 3D predicted model indicates that the $\mathrm{N}$-terminal domain between 1 and 21-24 amino acids might form a random dispersive domain.
This domain can attain various configurations but still be functional, and this domain might contribute toward receptor-mediated inhibition of Cav2.2 either by direct interaction or through an adaptor protein. The "random dispersive N-Terminal domain" still needs to be proven experimentally.

\section{CONCLUSION}

Dexras1 and RHES, both the GTP binding monomeric proteins have reported to influence many physiological processes and signaling mechanisms of many diseases. They both could be targeted as a potential pharmaceutical and therapeutic drug in future. The 3D structure of these two proteins will be helpful to know the interaction of these proteins. The 3D structure yet not described in any of the database. Our study for the prediction of 3D structure of these proteins by in silico method will be a step forward in knowing the interaction of these proteins with the other interactive proteins. This structural prediction might become the bases to know the exact molecular mechanisms involved in interactions and signaling of these two proteins, and it will also be helpful in further experimentation and hypothesizing the mechanism involved in signaling and modulation of physiological processes.

\section{ACKNOWLEDGMENT}

We would like to acknowledge Dr. Manish Kumar Gupta, Assistant Professor, Department of Bioinformatics, Chhatrapati Shahu Ji Maharaj University, Kanpur, for his help and suggestions for this analysis.

\section{AUTHORS' CONTRIBUTIONS}

All the authors have worked significantly for the preparation of Manuscript. Server and Software were used to generate data by Rashmi Verma under the Guidance of Dr. Ashish Thapliyal and Dr. Navin Kumar.

\section{CONFLICTS OF INTEREST}

All authors have none to declare.

\section{REFERENCES}

1. Kemppainen RJ, Behrend EN. Dexamethasone rapidly induces a novel ras superfamily member-related gene in AtT-20 cells. J Biol Chem 1996;273:3129-31

2. Cismowski MJ, Takesono A, Ma C, Lizano JS, Xie X, Fuernkranz H, et al. Genetic screens in yeast to identify mammalian non receptor modulators of G-protein signaling. Nat Biotechnol 1999;17:878-83.

3. Brogan MD, Behrend EN, Kemppainen RJ. Regulation of dexras1 expression by endogenous steroids. Neuroendocrinology 2001;74:244-50.

4. Spano D, Branchi I, Rosica A, Pirro MT, Riccio A, Mithbaokar P, et al. RHES is involved in striatal function. Mol Cell Biol 2004;24:5788-96.

5. Falk JD, Vargiu P, Foye PE, Usui H, Perez J, Danielson PE, et al. RHES: A striatal-specific ras homolog related to dexras1. J Neurosci Res 1999;57:782-8.

6. Cismowski MJ, Ma C, Ribas C, Xie X, Spruyt M, Lizano JS, et al. Activation of heterotrimeric G-protein signaling by a ras-related protein: Implications for signal integration. J Biol Chem 2000;275:23421-4.

7. Graham TE, Prossnitz ER, Dorin RI. Dexras1/AGS-1 inhibits signal transduction from the gi-coupled formyl peptide receptor to Erk-1/2 MAP kinases. J Biol Chem 2002;277:10876-82.

8. Thapliyal A, Verma R, Kumar N. Small G-Proteins dexras1 and RHES and their role in pathophysiological processes. Int J Cell Biol 2014;2014:308535.

9. Graham TE, Key TA, Kilpatrick K, Dorin RI. Dexras1/AGS-1, a steroid hormone-induced guanosine triphosphate-binding protein, inhibits 3,5-cyclic adenosine monophosphate-stimulated secretion in AtT-20 corticotroph cells. Endocrinology 2001;142:2631-40.

10. Thapliyal A, Bannister RA, Hanks C, Adams BA. The monomeric G-proteins AGS1 and RHES selectively influence G-i-dependent signaling to modulate N-type (Ca-2.2) calcium channels. Am J Physiol Cell Physiol 2008;295:C1417-26.

11. Ball GF, Balthazart J. Hormonal regulation of brain circuits mediating male sexual behavior in birds. Physiol Behav 2004;83:329-46.

12. McGrath MF, Ogawa T, de Bold AJ. Ras dexamethasone-induced Protein 1 is a modulator of hormone secretion in the volume overloaded 
heart. Am J Physiol Heart Circ Physiol 2012;302:H1826-37.

13. Subramaniam S, Snyder SH. Huntington's disease is a disorder of the corpus striatum: Focus on RHES (ras homologue enriched in the striatum). Neuropharmacology 2011;60:1187-92.

14. Vaidyanathan G, Cismowski MJ, Wang G, Vincent TS, Brown KD, Lanier SM. The ras-related proteinAGS1/RASD1 suppresses cell growth. Oncogene 2004;23:5858-63.

15. Sahare P, Moon A. In Silico modelling of B-Lactam resistant Enterococcus faecalis $\mathrm{Pbp} 4$ and its interactions with various phytoligands. Int J Pharm Pharm Sci 2016;8:151-5.

16. Gupta SK, Mandal P. Bioinformatic characterization of sod under uv in fenugreek (Family: Fabaceae). Int J Pharm Pharm Sci 2016;8:231-7.

17. Fang M, Jaffrey SR, Sawa A, Luo X, Ye K, Snyder SH. Dexras1: A G-protein specifically coupled to neuronal nitric oxide synthase via CAPON. Neuron 2000;28:183-93.
18. Chothia C, Lesk AM. The relation between the divergence of sequence and structure in proteins. EMBO J 1986;5:823-6.

19. Marti-Renom MA, Stuart AC, Fiser A, Sanchez R, Melo F, Sali A. Comparative protein structure modeling of genes and genomes. Annu Rev Biophys Biomol Struct 2000;29:291-325.

20. Schwede T, Kopp J, Guex N, Peitsch MC. SWISS-MODEL: An automated protein homology-modeling server. Nucleic Acids Res 2003;31:3381-5

21. Roy A, Kucukural A, Zhang Y. I-TASSER: A unified platform for automated protein structure and function prediction. Nat protoc 2010;5:725-38.

22. Roy A, Yang J, Zhang Y. COFACTOR: An accurate comparative algorithm for structure-based protein function annotation. Nucleic Acids Res 2012;40:W471-7. 